\title{
Evolución de la producción científica del Instituto Nacional de Técnica Aerospacial (1990-2005)
}

\author{
Silvia Paz Otero \\ Miguel Ángel Alonso Valdivieso \\ Fernando Mérida Martín \\ Instituto Nacional de Técnica Aeroespacial
}

\section{Resumen}

El Instituto Nacional de Técnica Aeroespacial (INTA) es el organismo público de referencia en la investigación aeronáutica y espacial desde su creación en 1942. A lo largo de su historia ha realizado multitud de actividades en el ámbito de la investigación básica, aplicada y de desarrollo, resultado de las cuales es su muy notable producción científica, que se ha traducido en la publicación de artículos en revistas científicas y técnicas, así como de monografías, en la colaboración en congresos y jornadas especializadas y también en la presentación de documentos adscritos a la denominada literatura gris, como patentes o normas.

El objetivo principal de este trabajo es analizar dicha producción durante el período 1990-2005 a través de un estudio cuantitativo y cualitativo: $a$ ) desde un punto de vista cuantitativo, se pretende identificar el volumen de las publicaciones del INTA, su impacto, el grado de colaboración del Instituto con otras instituciones, las materias de interés tratadas..., a través de la interrogación de bases de datos multisectoriales (por ejemplo, ISI); $b$ ) desde un punto de vista cualitativo, se intentará determinar los distintos patrones para la divulgación científica que siguen los investigadores del INTA en función de su formación, adscripción, origen o especialidad. Asimismo, se observará y reflexionará sobre la enorme diversidad de métodos utilizada para identificación de los autores, las instituciones, las revistas, etcétera, las distintas fuentes empleadas y el grado de dificultad que ello implica para la obtención y evaluación de los datos, que determina, en ocasiones, la elaboración de unas conclusiones fiables.

Palabras clave: Instituto Nacional de Técnica Aeroespacial. Indicadores bibliométricos. Producción científica. Evaluación científica. Colaboración científica.

\section{Abstract}

The National Institute of Aerospace Technique (INTA) is the establishment for aeronautics and space research since its creation in 1942. During more than sixty 
years of history, INTA has performed an enormous variety of activities in the basic, applied and development research. Result of this research process is a vast scientific production that has been expressed in the publication of monographs, articles in technical and scientific periodicals, and communications presented to specialized congresses or workshops as well. INTA has also produced a significant amount of grey literature in the form of standards, technical reports, memorandums, and other types of documents.

The main aim of this paper is to analyze this production during the period 1990-2005, through a quantitative and qualitative study: a) from a quantitative perspective, we would try to identify the amount of INTA publications, its impact, the degree of collaboration with other institutions, the disciplines covered... To get these data, multisector information search tools (ISI, for instance) are going to be used; $b$ ) from a qualitative perspective, and as result from the previous study, we will try to identify the different strategies that INTA scientists follow for their scientific spread, that vary depending his or their formation, adscription, origin or branch. Also, we will take into account the problems and difficulties found in the process for finding data because of the enormous diversity of forms to identify authors, institutions, publications or periodicals in the sources used. This situation implies added difficulties in the elaboration of reliable conclusions.

Key words: National Institute of Aerospace Techniques. Bibliometric indicators. Scientific production. Scientific evaluation. Scientific collaboration.

\section{Introducción}

El Instituto Nacional de Técnica Aeroespacial (INTA) es el organismo público de investigación aeronáutica y espacial español desde su fundación en 1942 como Instituto Nacional de Técnica Aeronáutica, y constituye el centro público de referencia para el desarrollo tecnológico y la investigación en su ámbito de actividad.

Actualmente el INTA está adscrito al Ministerio de Defensa y forma parte de la Red de Organismos Públicos de Investigación. Desde el mismo momento de su fundación, no solamente ha sido y sigue siendo laboratorio puntero para la investigación en las ramas de la ciencia más íntimamente ligadas al aeroespacio, sino que sus técnicos y especialistas contribuyen de manera notable al avance de otras disciplinas, como la física, la automoción, la astrofísica, la biología, el medio ambiente, la energía, etcétera.

El INTA ha participado desde los años cincuenta en multitud de proyectos propios, en iniciativas nacionales o internacionales, ha sido y es miembro de organismos europeos de investigación aeroespacial —en ocasiones incluso miembro fundador - y, en función de ello, ha participado en desarrollos fundamentales para el avance de la tecnología aeroespacial, facilitando el incremento y la mejora de 
la participación de la industria española en los grandes programas de investigación y desarrollo llevados a cabo en Europa.

Resultados tangibles de su actividad son los centenares de publicaciones en forma de monografías, artículos científicos y de divulgación, presentaciones o normas técnicas, entre otros elementos de diseminación del conocimiento y de la tecnología.

A lo largo de la dilatada historia del INTA el grado de producción científica ha sufrido fluctuaciones y variaciones diversas, producto de las cambiantes circunstancias a las que se ha visto sometido. Durante un largo periodo de tiempo se configuró como la única referencia a nivel nacional, funcionando como un laboratorio institucional que facilitó la formación de técnicos de altísimo nivel que, más tarde, pasaron a formar parte de los cuadros dirigentes de la empresa privada o de la Universidad. Estos grandes especialistas produjeron una ingente y en extremo valiosa colección de monografías, aún obras de consulta básica para la formación de nuevos ingenieros.

Posteriormente, la producción editorial del INTA fue mermando, fundamentalmente por el cambio de dirección estratégica de la institución, que fue decantándose de un modo notable por el desarrollo tecnológico más que por la investigación básica. Aun así, han seguido apareciendo un buen número de trabajos científicos en publicaciones nacionales e internacionales, pero la difusión del conocimiento adquirido ha ido en direcciones distintas a la publicación de trabajos en revistas científicas, teniendo más peso la producción de informes técnicos y otro tipo de literatura gris, no fácilmente visible utilizando medios como los usados en este estudio.

A partir de los años noventa del siglo XX, el INTA volvió a impulsar su orientación científica, dando nuevamente relevancia a la investigación básica, sin dejar de mantener su perfil tecnológico, a partir de la incorporación a su estructura de dos centros directamente ligados a las tareas de la astrofísica, la física fundamental y la astrobiología. En 1991 se creó el Laboratorio de Astrofísica y Física Fundamental (LAEFF), iniciativa que conllevó el establecimiento de acuerdos de cooperación con el Consejo Superior de Investigaciones Científicas (CSIC) y con la European Space Agency (ESA), con el objeto fundamental de contribuir desde el INTA al incremento de la investigación y explotación científica de los recursos de los departamentos técnicos y de las instalaciones espaciales del Instituto. En 2000 se creó el Centro de Astrobiología, que también es una experiencia en colaboración estrecha con el CSIC y con la NASA. El Centro de Astrobiología (CSIC-INTA) centra su atención en estudios experimentales y teóricos fundamentales para tratar de dilucidar cómo se originó y ha evolucionado la vida en nuestro planeta. Estos estudios son básicos para comprender cómo ha podido surgir y distribuirse la vida en el universo.

Scire. 12 : 2 (jul.-dic. 2006) 119-135. ISSN 1135-3716. 
Teniendo en cuenta lo anterior, hemos considerado pertinente analizar de un modo cuantitativo y cualitativo la producción científica de estos tres organismos de investigación y estudiar los distintos comportamientos que, durante un periodo dilatado y reciente en el tiempo, han mostrado sus científicos.

Este trabajo puede servir de base para otros posteriores que aporten datos interesantes para la política científica de la institución y para la identificación de posibles grupos de excelencia, faciliten la determinación de la posición del INTA en el contexto internacional de la investigación aeroespacial e identifiquen los grupos de investigación más activos a nivel micro.

\section{Metodología}

El presente análisis surge de la inquietud por conocer la producción científico-técnica del INTA a nivel global. En la actualidad, el Instituto está integrado por varias dependencias. En este estudio se identifican tres núcleos: $a$ ) el INTA como organismo central; $b$ ) el Laboratorio de Astrofísica y Física Fundamental (LAEFF); c) el Centro de Astrobiogía (CAB). El primero de ellos comprende las instalaciones siguientes: el campus de Torrejón de Ardoz, en donde se ubica la mayor parte del Instituto; el CEDEA, ubicado en El Arenosillo, Huelva; las estaciones espaciales de Villafranca del Castillo y de Maspalomas.

Para realizar este estudio nos hemos basado en la base de datos del Institute for Scientific Information (ISI). Esta base de datos es multidisciplinar, cubre las ciencias exactas, médicas y naturales, y recoge 5683 revistas categorizadas en 68 temas. El período analizado comprende los años 1990-2005 y para él se han procesado un total de 798 referencias dentro de las bases de datos SCI (Science Citation Index), IC (Index Chemicus) y Current Chemical Reactions (CCR-Expanded). Estas referencias se han obtenido a través de las búsquedas en el campo "AD" (address), tratando de seleccionar los registros de las tres entidades analizadas (INTA, LAEFF, CAB). El proceso no ha estado exento de complicaciones, puesto que no existe uniformidad en la adscripción de los autores a la organización de la que dependen.

Previamente a la constatación de nuestros resultados, se hace necesario introducir una serie de puntualizaciones: $a$ ) se ha partido siempre de la información extraída de las bases de datos anteriormente mencionadas. No se ha consultado ninguna otra fuente de información; $b$ ) se han empleado estrategias de búsqueda independientes para cada una de las organizaciones, con el fin de aislar totalmente cada uno de los datos; $c$ ) se ha constatado que existen distintas variaciones a la hora de consignar los nombres de las organizaciones: INTA: INTA, inst nacl tecn aerosespacial, natl inst aerosp technol; LAEFF: LAEFF, lab space astrophys, astrofis espacial \& fis fundamental lab, astrofis espacia \& fis fundamental lab; CAB: CAB, ctr astrobiol, CSIC-INTA, NASA-INTA. 
Del mismo modo, se ha comprobado cómo existen organizaciones cuyos acrónimos son coincidentes con los que nosotros identificábamos como propios: $a$ ) tenemos tres institutos cuyas siglas son INTA, pero que no corresponderían a nuestro Instituto de referencia, y que se localizan Granada (España), en Argentina y en Chile; $b$ ) no se han constatado homonimias en los acrónimos para el caso del LAEFF; $c$ ) para el caso del caso del CAB, podemos mencionar la existencia de un centro en Italia que se identifica con estas mismas siglas.

Asimismo, existen autores que a pesar de estar vinculados orgánicamente a la organización matriz (INTA), no firman sus contribuciones como pertenecientes a ella. Nos referimos a los que desarrollan su labor investigadora en la Estación Experimental de El Arenosillo, situada en Huelva pero que es un departamento más del INTA.

Siguiendo con el tema de las organizaciones, las bases de datos del ISI no se han mostrado infalibles a la hora de referenciar los datos, puesto que hemos constatado fallos en la introducción de los nombres: por ejemplo, LAEEF, Lab Astofis, LAEF.

En cuanto a la colaboración, los autores se han contabilizado de forma individual para los datos globales, pero los artículos se consideran únicos.

Para el análisis de las temáticas nos hemos basado en la clasificación en áreas y disciplinas que propone el propio ISI.

\section{Datos obtenidos}

\subsection{Evolución de la productividad por años}

\section{Datos globales}

Se han obtenido un total de 798 referencias para el período 1990-2005, repartidas del siguiente modo: INTA: 131 registros (período 1990-2005); LAEFF: 424 registros (período 1991-2005); CAB: 243 registros (período 2000-2005).

El número medio para el total es de 53 contribuciones por año. Solo el LAEFF y el CAB logran superar en algún año el valor establecido como media. El LAEFF es el centro que registra el valor máximo de contribuciones por año (1999, con 58 contribuciones) y el INTA el que registra el valor mínimo ( 2 contribuciones en los años 1992, 1993 y 1996).

Si prestamos atención al total registrado para las tres entidades, el año más productivo es 2004, con 102 contribuciones; y el menos productivo, con 69 artículos, 2000. Para constatar estos valores se han tenido en cuenta únicamente los años en los que las tres aportan datos, es decir, solo a partir de la fecha de creación del CAB, el último centro en incorporarse al INTA. 


\begin{tabular}{|c|c|c|c|c|}
\hline Año & INTA & LAEFF & CAB & Total \\
\hline 1990 & 3 & 0 & 0 & 3 \\
\hline 1991 & 0 & 5 & 0 & 5 \\
\hline 1992 & 2 & 14 & 0 & 16 \\
\hline 1993 & 2 & 16 & 0 & 18 \\
\hline 1994 & 7 & 13 & 0 & 20 \\
\hline 1995 & 7 & 20 & 0 & 27 \\
\hline 1996 & 2 & 26 & 0 & 28 \\
\hline 1997 & 5 & 40 & 0 & 45 \\
\hline 1998 & 6 & 52 & 0 & 58 \\
\hline 1999 & 17 & 58 & 0 & 75 \\
\hline 2000 & 11 & 36 & 22 & 69 \\
\hline 2001 & 13 & 36 & 35 & 84 \\
\hline 2002 & 16 & 32 & 25 & 73 \\
\hline 2003 & 13 & 33 & 54 & 100 \\
\hline 2004 & 16 & 31 & 55 & 102 \\
\hline 2005 & 11 & 12 & 52 & 75 \\
\hline Total & 131 & 424 & 243 & 798 \\
\hline
\end{tabular}

Tabla I. Producción por año de publicación $(I N T A+L A E F F+C A B)$.

Comparando las tres entidades, se puede apreciar cómo el INTA ofrece los peores resultados de productividad, porque, pese a ser la institución más longeva en el tiempo (se crea en 1942, lo que le supone en el año 1990 un trayectoria investigadora asentada), es la que menores contribuciones aporta. Por el contrario, el CAB, siendo la institución más joven (se crea en el año 2000), ofrece el mejor balance productivo.

Tal y como se aprecia en el gráfico de la figura 1, la producción científica global ha ido en aumento desde su aparición, por lo que es posible constatar la consolidación global del INTA como institución investigadora. La línea de evolución general no ofrece demasiadas variaciones en comparación con la evolución de las tres entidades. Se aprecian oscilaciones entre valores al alza y al descenso, con variaciones poco destacadas salvo en los casos ya apuntados.

Se observa la disparidad entre la tendencia temporal de los tres organismos; por un lado, el LAEFF presenta la caída más pronunciada, mientras que INTA y CAB mantienen unos parámetros regulares, obedeciendo el descenso en el primero a algunas consideraciones que serán posteriormente apuntadas en el análisis independiente de esta institución. 


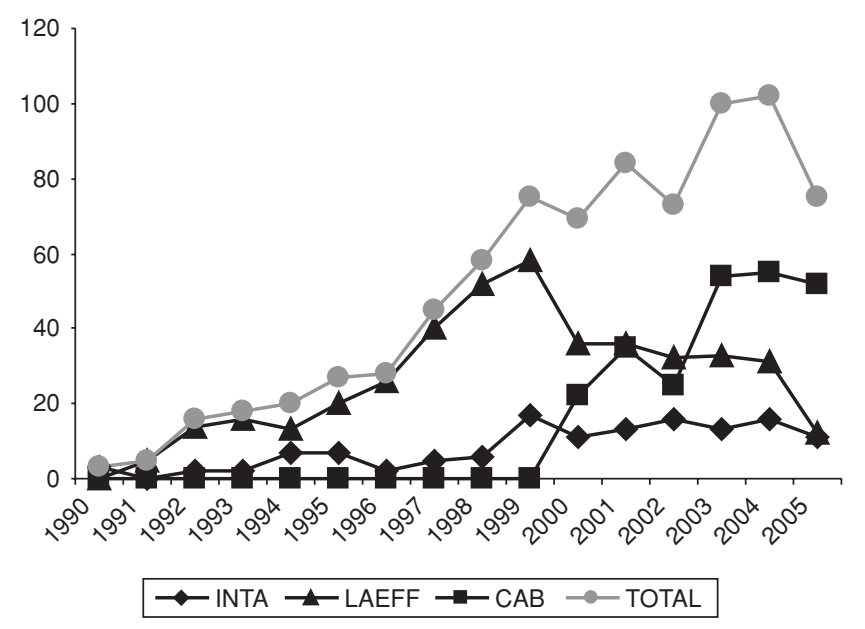

Figura 1. Evolución temporal de la producción de las tres entidades en relación con la evolución general.

Pese al ligero descenso en el número de contribuciones en el año 2005, se trata de un descenso relativo, puesto que parece claramente motivado por la acusada disminución del número de contribuciones facilitadas por una de las tres entidades analizadas. En las otras dos, esa reducción es mucho menos destacada, no apuntándose claramente una tendencia hacia el descenso.

Si analizamos cada una de los centros por separado, las conclusiones obtenidas son bastantes dispares:

1. El número total de contribuciones registradas para el caso del INTA, considerado como institución única, es de 131, distribuidas según el número establecido en la tabla II. Los valores registrados corresponden al total del período analizado, 1990-2005. El número medio de contribuciones es de 8,7, es decir, casi 9 publicaciones por año. El año más productivo es 1999, con 17 contribuciones, un valor que casi duplica la media establecida. Por el contrario, hay un año, 1991, en el que no se registra ninguna contribución.

Es necesario destacar que a partir de 1999 se constata una tendencia hacia el crecimiento, con valores casi siempre ligeramente superiores al establecido como media. Esto se explica por la aparición del Centro de Astrobiología, que fue inaugurado a principios de 2003, si bien había comenzado su actividad a mediados de 1999, siendo el año 2000 el punto de inflexión que vemos en la figura 3 , que coincide con las primeras publicaciones del $\mathrm{CAB}$. 
2. El LAEFF se creó en mayo del año 1990, por lo que los primeros valores registrados corresponden al año 1991. El número total de contribuciones contabilizadas para el período 1991-2005 es de 424, recogiéndose como valor medio 30 contribuciones por año.

El año más productivo es 1999, con 58 contribuciones; y el menos, 1991, con 5. Hay que recordar que en 1991 el LAEFF solo llevaba en funcionamiento un año, por lo que todavía no había enraizado la actividad investigadora, que fructificaría de forma notable en torno al final de los años noventa, cuando estaban trabajando en el LAEFF numerosos y activos grupos de investigación, grupos que se disolvieron o vieron mermados el número de sus miembros a partir de 2002, lo cual se tradujo en un descenso en el número de contribuciones, situación producida por la marcha de los investigadores a otros centros.

El gráfico de la figura 1 parece iniciarse con una tendencia hacia el crecimiento hasta el año 1999, por las razones anteriormente apuntadas. Posteriormente, se asienta en torno a valores cercanos al establecido como valor medio. Un nuevo descenso vuelve a manifestarse de forma mucho más acusada que en el caso anterior a partir del año 2005. Las razones que se esgrimen para ese descenso son nuevamente las escasas posibilidades del INTA para la retención del personal investigador una vez que ha concluido su período como becarios pre y posdoctorales. De hecho, en la actualidad el LAEFF cuenta solo con un investigador contratado, siendo el resto del personal becarios de investigación. La falta de estabilidad laboral se apunta en este caso como causa directamente vinculada al descenso en el número de publicaciones.

3 El CAB se creó en 1999. Sus primeros valores significativos aparecen en el año 2000. El número total de contribuciones en el período comprendido entre 2000 y 2005 es de 243, con un promedio anual de 40,5 publicaciones.

El año más productivo es 2004 con 55 publicaciones; el menos, 2000, con 22. En el gráfico de la figura 1 podemos observar una tendencia positiva al crecimiento. Los valores se estabilizan por encima de 50 publicaciones a partir de 2003, año en que se inauguraron las instalaciones y se dotó a los laboratorios de plena capacidad operativa. 


\subsection{La colaboración científica}

\subsubsection{Datos globales}

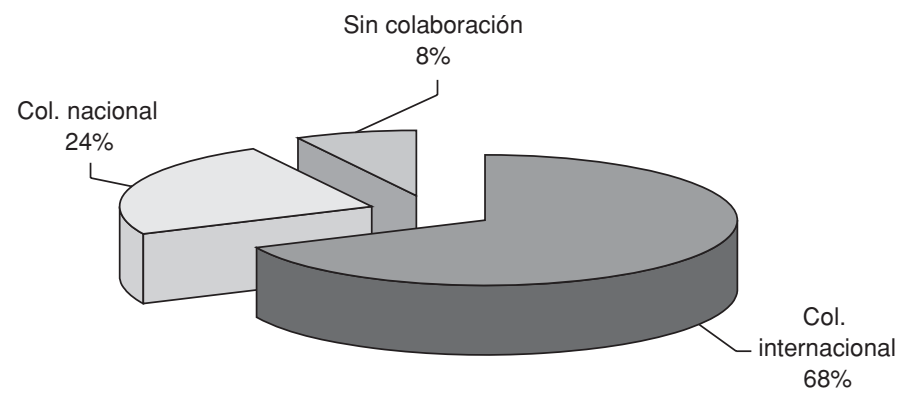

Figura 2. Tipos de colaboración $(I N T A+L A E F F+C A B)$.

Los patrones de colaboración demuestran un alto nivel tanto nacional como internacional, ya que los trabajos sin colaboración representan solo un $8 \%$ del total, frente al $68 \%$ que suponen los de colaboración internacional y el 24\% los de colaboración nacional.

Un estudio de Isabel Gómez Caridad y su equipo del CINDOC (2004) revela que en España el índice de colaboración internacional es del 33\%. Arurachalam y Doss (2000) sostienen la importancia que tiene hoy día la colaboración entre científicos, que se apoyan mutuamente para la generación de nuevo conocimiento y buscan por otra parte la formación de redes de trabajo en su área con el objetivo de alcanzar una mayor visibilidad y reconocimiento en la comunidad científica a que pertenecen. Morillo, Bordons y Gómez (2000) señalan que la colaboración internacional ayuda a aumentar el crédito científico, el impacto y la aceptación.

\begin{tabular}{|l|c|c|c|c|}
\hline \multicolumn{2}{|c}{ Col. internacional } & Col. nacional & Sin colaboración & Total \\
\hline INTA & 59 & 51 & 21 & 131 \\
\hline LAEFF & 339 & 63 & 22 & 424 \\
\hline CAB & 144 & 79 & 20 & 243 \\
\hline Total & 542 & 193 & 63 & 798 \\
\hline
\end{tabular}

Tabla II. Tipos de colaboración (INTA + LAEFF + CAB). 


\subsubsection{Análisis del INTA}

\begin{tabular}{|l|c|c|c|c|}
\hline Año & Col. internacional & Col. nacional & Sin colaboración & Total \\
\hline 1990 & 0 & 2 & 1 & 3 \\
\hline 1991 & 0 & 0 & 0 & 0 \\
\hline 1992 & 1 & 0 & 1 & 2 \\
\hline 1993 & 1 & 0 & 1 & 2 \\
\hline 1994 & 5 & 2 & 0 & 7 \\
\hline 1995 & 4 & 2 & 1 & 7 \\
\hline 1996 & 1 & 1 & 0 & 2 \\
\hline 1997 & 3 & 1 & 1 & 5 \\
\hline 1998 & 2 & 4 & 0 & 6 \\
\hline 1999 & 5 & 11 & 1 & 17 \\
\hline 2000 & 7 & 2 & 2 & 13 \\
\hline 2001 & 7 & 2 & 4 & 16 \\
\hline 2002 & 7 & 8 & 1 & 13 \\
\hline 2003 & 7 & 3 & 3 & 16 \\
\hline 2004 & 7 & 7 & 2 & 11 \\
\hline 2005 & 2 & 6 & 3 & 131 \\
\hline Total & 59 & 51 & 21 & \\
\hline
\end{tabular}

Tabla III. Tipos de colaboración (INTA).

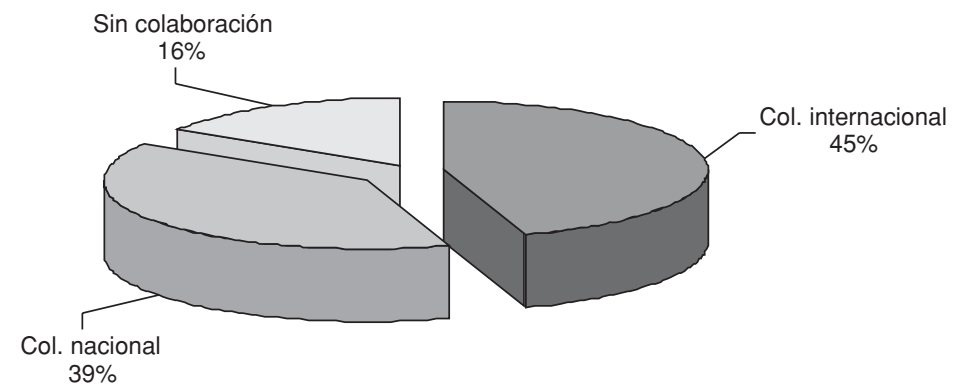

Figura 3. Tipos de colaboración (INTA). 


\subsubsection{Análisis del LAEFF}

\begin{tabular}{|l|c|c|c|c|}
\hline Año & Col. internacional & Col. nacional & Sin colaboración & Total \\
\hline 1991 & 2 & 3 & 0 & 5 \\
\hline 1992 & 9 & 4 & 1 & 14 \\
\hline 1993 & 14 & 1 & 1 & 16 \\
\hline 1994 & 11 & 1 & 1 & 13 \\
\hline 1995 & 9 & 9 & 2 & 20 \\
\hline 1996 & 23 & 1 & 2 & 26 \\
\hline 1997 & 30 & 5 & 5 & 40 \\
\hline 1998 & 39 & 11 & 2 & 52 \\
\hline 1999 & 44 & 11 & 3 & 58 \\
\hline 2000 & 30 & 5 & 1 & 36 \\
\hline 2001 & 34 & 1 & 1 & 36 \\
\hline 2002 & 31 & 1 & 0 & 32 \\
\hline 2003 & 29 & 4 & 0 & 33 \\
\hline 2004 & 22 & 6 & 3 & 31 \\
\hline 2005 & 12 & 0 & 0 & 12 \\
\hline Total & 339 & 63 & 22 & 424 \\
\hline
\end{tabular}

Tabla IV. Tipos de colaboración (INTA).

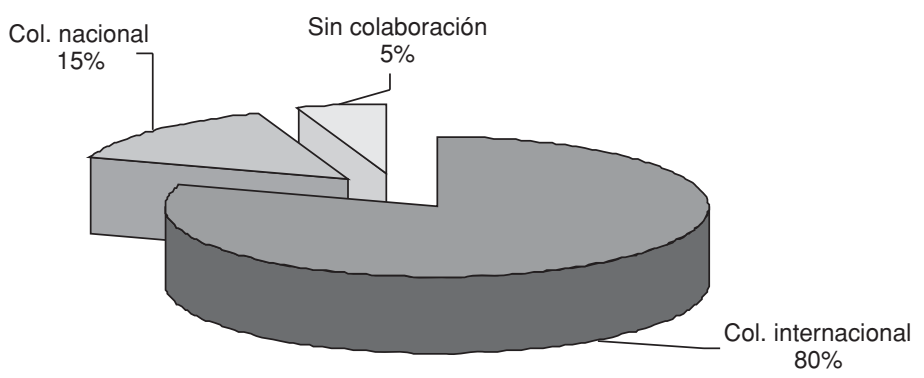

Tabla 4. Tipos de colaboración (INTA). 


\subsubsection{Análisis del $C A B$}

\begin{tabular}{|l|c|c|c|c|}
\hline Año & Col. internacional & Col. nacional & Sin colaboración & Total \\
\hline 2000 & 12 & 9 & 1 & 22 \\
\hline 2001 & 20 & 10 & 5 & 35 \\
\hline 2002 & 14 & 9 & 2 & 25 \\
\hline 2003 & 30 & 19 & 5 & 54 \\
\hline 2004 & 35 & 16 & 4 & 55 \\
\hline 2005 & 33 & 16 & 3 & 52 \\
\hline Total & 144 & 79 & 20 & 243 \\
\hline
\end{tabular}

Tabla V. Tipos de colaboración (CAB).

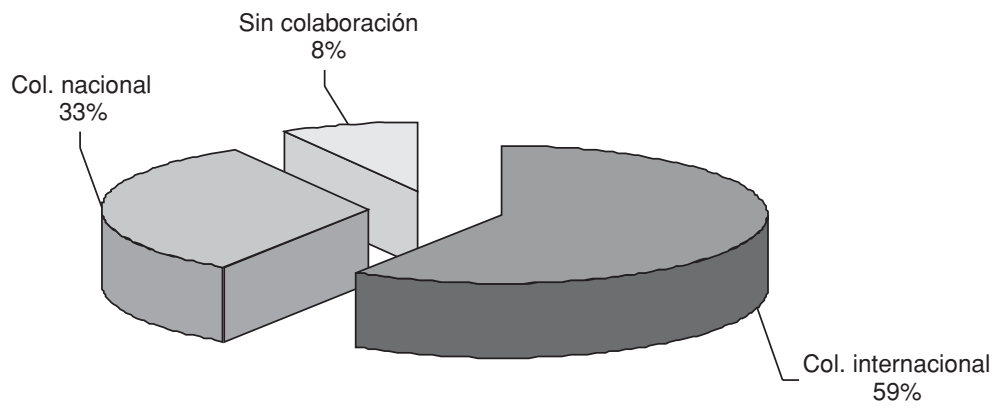

Figura 5. Tipos de colaboración (CAB).

\subsection{Clasificación temática}

La clasificación en grandes áreas y, dentro de ellas, en disciplinas, se ha realizado siguiendo los criterios temáticos impuestos por el ISI en todas sus bases de datos.

Es necesario hacer constar que en el caso del INTA se han recogido 11 referencias que no incluían datos en el campo "Topic" (materia). En el LAEFF se registran 27 referencias sin datos. Todos los registros del CAB contienen datos en el campo que estamos analizando.

\subsubsection{Datos totales de las áreas temáticas}

Siguiendo la clasificación en áreas y disciplinas de las bases de datos del ISI, las referencias analizadas quedarían adscritas a las siguientes grandes categorías: "Agricultura, biología y medio ambiente", "Biomedicina", "Física", "Ingeniería, tecnología", "Matemáticas", "Multidisciplinar" y "Química". 


\begin{tabular}{|l|r|r|r|r|r|}
\hline Área & \multicolumn{1}{|c|}{ INTA } & \multicolumn{1}{c|}{ LAEFF } & CAB & \multicolumn{1}{c|}{ Total } & $\%$ \\
\hline Agricultura, biología y medio ambiente & 6 & & 22 & 28 & 3 \\
\hline Biomedicina & 6 & & 46 & 52 & 6 \\
\hline Física & 82 & 398 & 111 & 591 & 73 \\
\hline Ingeniería, tecnología & 66 & & 22 & 88 & 11 \\
\hline Matemáticas & & & 9 & 9 & 1 \\
\hline Multidisciplinar & 3 & 11 & 22 & 36 & 4 \\
\hline Química & 9 & & 8 & 17 & 2 \\
\hline
\end{tabular}

Tabla VI. Áreas temáticas.

El área temática en la que se registran más trabajos es "Física". La diferencia en número de contribuciones con respecto al resto de las temáticas es abismal; salvo en el área mencionada, no se superan las 100 contribuciones. Aislando los resultados a un nivel de análisis independiente obtenemos el mismo balance: la mayor parte de los trabajos recopilados se publican en la categoría "Física". En el caso del INTA, la diferencia entre las dos áreas más productivas no es excesivamente destacable, algo que sí sucede para las otras dos entidades. El LAEFF se muestra excesivamente especializado, lo que contrasta con la tendencia multidisciplinar del $\mathrm{CAB}$, presente en todas las áreas en mayor o en menor medida.

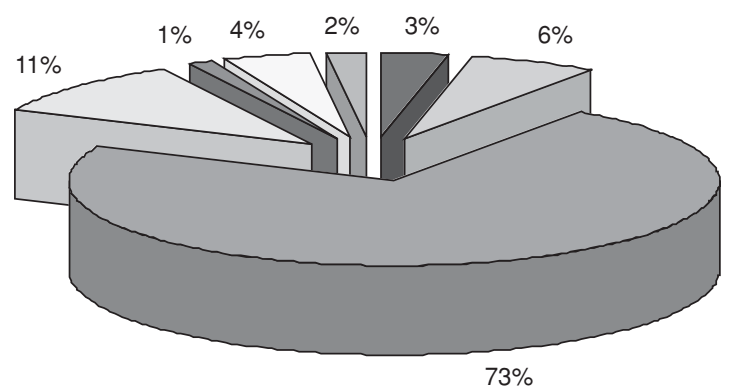

\begin{tabular}{|ll|}
\hline$\square$ Agricultura, biología y medio ambiente & $\square$ Biomedicina \\
$\square$ Física & $\square$ Ingeniería, tecnología \\
$\square$ Matemáticas & $\square$ Multidisciplinar \\
$\square$ Química & \\
\hline
\end{tabular}

Figura 6. Áreas temáticas. 


\subsubsection{Datos totales de las disciplinas temáticas}

Las disciplinas más productivas (citadas a partir de 10 referencias) son

- Física. Astronomía y astrofísica: 411 referencias

- Física. Física, multidisciplinar: 38 referencias

- Física. Geociencias, multidisciplinar: 33 referencias

- Biomedicina. Bioquímica y biología molecular: 29 referencias

- Física. Física, partículas y campos: 26 referencias

- Física. Meteorología y ciencia atmosférica: 23 referencias

- Física. Geoquímica y geofísica: 19 referencias

- Física. Física, estado sólido: 12 referencias

- Ingeniería, tecnología. Ingeniería aeroespacial: 10 referencias

\subsubsection{Datos por áreas, aislando los datos de las tres entidades}

\subsubsection{Agricultura, biología y medio ambiente}

Solo el INTA y el CAB presentan contribuciones en esta categoría, aunque la participación del primero es bastante menor; destaca el grupo de investigación en el campo de las energías renovables, que supone prácticamente el total de las contribuciones del INTA en esta área.

El medio ambiente, la biología y la biotecnología son las tres disciplinas en las que más artículos se adscriben dentro de esta categoría. Es el CAB la institución que, debido a las investigaciones que realiza, más registros acumula.

\subsubsection{Biomedicina}

Es el CAB la institución que más registros recopila en la categoría "Biomedicina", en parte gracias a los 26 que se adscriben a la disciplina de la bioquímica y biología molecular, que suponen más de la mitad de las contribuciones que el $\mathrm{CAB}$ registra en esta especialidad, y la mitad si comparamos este resultado con los valores globales. Esos valores globales se incrementan con las 6 contribuciones que aporta el INTA, puesto que el LAEFF no presenta ninguna en esta categoría.

\subsubsection{Física}

Es en la categoría "Física" donde se recogen más contribuciones a nivel global. Esto no debe sorprender, ya que estamos hablando de tres entidades que desarrollan buena parte de su labor investigadora en los campos de la astronomía y de la astrofísica, disciplinas que se encuadran dentro de la mencionada categoría. De facto, las contribuciones adscritas a ellas suponen casi el 70\% de los valores totales del área, y se aúpan también como las disciplinas donde más contribuciones se registran por institución. Es en el LAEFF donde esta proporción alcanza una dimensión mayor, llegando al 80\% de la producción total del centro. 
Otro de los grupos temáticos más destacados es el de las contribuciones que dentro de esta categoría llevan la etiqueta de física. Suponen un 15\% del total dentro de esta área, inmediatamente después de las contribuciones de astronomía y astrofísica. La participación del LAEFF en este sentido es muy destacable, con 53 contribuciones, cifra no muy lejana a las 36 que aporta el CAB. El INTA se mantiene en una discreta tercera posición, con cifras muy distantes de las anteriores. No obstante, sí hay que mencionar los 21 artículos que el INTA aporta dentro de la disciplina de la meteorología y ciencia atmosférica, merced a las aportaciones del grupo de investigación atmosférica que desarrolla su actividad en el Instituto.

\subsubsection{Ingeniería, tecnología}

Es en la categoría "Ingeniería y tecnología" donde el INTA obtiene sus mejores resultados en relación con el número global de publicaciones: 26 contribuciones estarían vinculadas al campo de la ingeniería y 12 al de la investigación dedicada al estudio de materiales. Ambos campos de investigación suponen más de la mitad de las contribuciones que el INTA arroja en esta categoría temática, en la que destaca nuevamente la ausencia del LAEFF, por las peculiaridades investigadoras ya apuntadas.

Por su parte, el CAB destaca por las contribuciones que aporta en la disciplina de informática: 17 de un total de 22.

\section{Conclusiones}

En sintonía con algunos trabajos anteriores del CINDOC concluimos que la ciencia aplicada tiene un carácter local y aparece mejor representada en otras bases de datos, que en nuestro caso puede ser ICYT. También constatamos que podría ser más útil la utilización de bases de datos especializadas, como por ejemplo ADS (Astrophysics Data System).

El INTA aparece peor representada con respecto a las otras dos entidades, interpretamos que por su mayor perfil tecnológico, siguiendo el argumento anterior.

El número total de contribuciones es de 798; se registra como valor medio 53 por año y puede apreciándose una tendencia hacía el crecimiento.

El LAEFF es la entidad más productiva a nivel global, pero es el CAB el que arroja un mejor balance productivo.

Los patrones de colaboración son muy elevados, sobre todo en el caso de la internacional. Asimismo, existe escaso interés por establecer redes de colaboración institucional, más allá de las evidentes con el CSIC.

La disciplina más productiva es la que se identifica como "Astronomía y astrofísica".

Se constata que un gran número de trabajos aparecen encuadrados dentro de la categoría "Física ", siendo contribuciones que identificamos dentro de la materia 
de la astronomía, por lo cual consideramos que hay una inapropiada ubicación de categorías, al menos en este caso.

El motor de búsqueda no está lo suficientemente perfeccionado para filtrar selecciones complejas, al menos en el campo "AD" (address).

Finalmente, no existe unanimidad en la adscripción de los autores a sus entidades de referencia.

\section{Referencias}

Arunachalam, S.; Jinandra Doss, M. (2000). Science in a small country at a time of globalization: domestic and international collaboration in new biology in Israel. // Journal of Information Science. 51:1 (2000) 39-49.

Gómez, Monique (2002). A bibliometric study to manage a journal collection in an astronomical library: some results. // Corbin, B.; Bryson, E.; Wolf, M. (eds.). Library and Information Science in Astronomy IV. Praga, 2002. 214-222.

Leta, Jacqueline; Lewison, Grant (2003). The contribution of women in Brazilian science: a case study in astronomy, immunology and oceanography. // Scientometrics. 57:3 (2003) 339-353.

Llana Martín, Amelia de la; Barredo Sobrino, M. ${ }^{a}$ Pilar (2003). Estudio bibliométrico de la producción científica de los departamentos básicos de la Facultad de Medicina de la Universidad Autónoma de Madrid (1999-2000). http://biblioteca.uam.es/documentos/ cc10.pdf (2006-03-22).

López López, Pedro (1996). Introducción a la bibliometría. Valencia: Promolibro, 1996.

López Piñero, J. M.; Terrada, M. L. (1992). Los indicadores bibliométricos y la evaluación de la actividad médico-científica (I): usos y abusos de la bibliometría. // Medicina Clínica. 98 (1992) 64-68.

López Piñero, J. M.; Terrada, M. L. (1992). Los indicadores bibliométricos y la evaluación de la actividad médico-científica (III): los indicadores de producción, circulación y dispersión, consumo de la información y repercusión. // Medicina Clínica. 98 (1992) 142-148.

López Piñero, J. M.; Terrada, M. L. (1992). Los indicadores bibliométricos y la evaluación de la actividad médico-científica (IV): la aplicación de los indicadores. // Medicina Clínica. 98 (1992) 384-388.

Maltrás Barba, Bruno (2003). Los indicadores bibliométricos. Fundamentos y aplicación al análisis de la ciencia. Gijón: Trea, 2003.

Morillo Ariza, F.; Bordons, M.; Gómez, I. (2000). An approach to interdisciplinary trough bibliometric indicators. Scientometrics. 51:1 (2000) 203-222.

Rojo, Raquel; Gómez, Isabel (2006). Analysis of the Spanish scientific and technological output in the ICT sector. // Scientometrics. 66:1 (2006) 101-121.

Rovira, Lluís; Senra, Pau; Jou, David (2000). Bibliometric analysis of physics in Catalonia: Towards quality consolidation? // Scientometrics. 49:2 (2000) 233-256.

Sánchez, S. F.; Benn, C. R. (2004). Impact of astronomical research from different countries. // Astronomische Nachrichten. 325:5 (2004) 445-450.

Scire. 12 : 2 (jul.-dic. 2006) 119-135. ISSN 1135-3716. 
Sánchez Ron, José Manuel (1997). INTA: 50 años de ciencia y técnica aeroespacial. Madrid: Ministerio de Defensa, 1997.

Schulman, E.; French, James C.; Powell, Allison L.; Eichhorn, G.; Kurtz, M. J.; Murray, S. S. (1997). Trends in astronomical publication between 1975 and 1996. // Publications of the Astronomical Society of the Pacific. 109 (Nov. 1997) 1278-1284.

Trueba, Frank J.; Guerrero, Héctor. (2004). A robust formula to credit authors for their publications. // Scientometrics. 60:2 (2004) 181-204. 